\title{
5-aminolevulinic acid (5-ALA) fluorescence-guided Mohs surgery resection of penile-scrotal extramammary Paget's disease
}

\author{
Xiaoqiong Peng ${ }^{\S}$, Wei Qian ${ }^{\S}$ Jiangang Hou* \\ Department of Urology Research Institute, Huashan Hospital Affiliated to Fudan University, Shanghai, China.
}

\begin{abstract}
Summary This report aims to evaluate the usefulness of 5-aminolevulinic acid (5-ALA) fluorescenceguided Mohs surgery resection of penile-scrotal extramammary Paget's disease for achieving maximum tumor resection. Between January 2014 and December 2015, 5 patients underwent surgical resection of a penile-scrotal extramammary Paget's disease in department of urology, Huashan hospital, Fudan University. All patients were coated with 5-ALA (concentration of $20 \%$ ) throughout the scrotum and the visible range of the lesion plus a $2 \mathrm{~cm}$ margin 3 hours before the induction of anesthesia. 5-ALA fluorescence was visualized using an ultraviolet (UV) light at $405 \mathrm{~nm}$. Surgical margin was determined in a standardized manner. The extent of resection was evaluated on the basis of frozen and histology sections. If the fluorescence positive punctate lesions were found outside the resection range, we removed the lesions and sent them for pathological examination. All data were prospectively collected, and the shortand long-term outcomes of the treatment strategy were analyzed. Lesions in the blue light turns red after irradiation, the fluorescence-guided surgery delineated range is less than the naked eye, intraoperative frozen prompted negative margins, postoperative pathological diagnosis. A total of $\mathbf{3 1}$ scattered lesions were found. After biopsy pathology prompted four were positive. In conclusion, 5-ALA fluorescence-guided minimum range can be completely removed in penile-scrotal Paget's lesions, and it is able to detect distant scattered lesions.
\end{abstract}

Keywords: 5-aminolevulinic acid, fluorescence, Mohs surgery, extramammary Paget's disease

\section{Introduction}

Extramammary Paget's disease (EMPD) is a rare malignancy that mainly affects the anogenital region in elderly people, first described by Crocker in 1889 (1). The disease is usually misdiagnosed as eczema and the lesions commonly develop in the vulva, penis, scrotum, perineum, perianal area, umbilicus and axilla.

The method of surgical excision and defining the surgical margin of EMPD remain controversial. At present, surgical modalities including Mohs micrographic surgery, fluorescent dyes and frozen section examination (FSE) are recommended to ensure clear margins. With regard to treatment, neither the

Released online in J-STAGE as advance publication October $16,2017$.

${ }^{\S}$ These authors contributed equally to this work.

*Address correspondence to:

Dr. Jiangang Hou, Department of Urology Research Institute, Huashan Hospital Affiliated to Fudan University, Shanghai, 200040, China.

E-mail: hou_jiangang@126.com method of surgical excision nor the size of the surgical margin has been standardized because of the anatomical complexity of genital lesions. Even extensive resections are complicated by a high local recurrence rate (15$44 \%$ ) due to several characteristics of EMPD, such as multicentricity and ill-defined margins (2-3).

While many attempts to increase the rate of complete resection have been made, conclusive evidence supporting the efficacy of these approaches is limited. Studies using photodynamic therapy have been published, however achieving solely palliative results (4). Imiquimod cream at 5\% concentration, 5\% 5-fluorouracil cream, $\mathrm{CO}_{2}$ laser and the association of two or more therapeutic approaches may also be used, with variable results (5). Increasing the incidence of complete resection, without causing excess morbidity, requires new methods to accurately identify neoplastic tissue intraoperatively, such as use of the drug 5-aminolevulinic acid (5-ALA). It is utilized as a precursor of a photosensitizer for performing a photodynamic diagnosis (PDD) and photodynamic therapy (PDT) to confirm and kill tumor cells (6-8). In 
addition to the utilization of PDD and PDT, 5-ALA has been used to treat inflammatory disease, autoimmune disease and transplantation due to the anti-inflammation and immunoregulation properties by upregulation of heme oxygenase (HO)-1 expression and release of heme metabolites $(9,10)$. Monitoring the distribution of PpIX is a useful tool for visualizing tumor locations, since the ALA-induced PpIX fluorescence accumulates primarily in malignant tumor tissue and its fluorescence is much stronger than that in normal skin. However, the clinical demarcation of the lesion is still a frequent problem. 5-ALA has been widely used to aid intraoperative identification of malignant gliomas, showing better cytoreduction and marked benefits for patients (11). Chan et al reported that 5-ALA is a useful intraoperative guide for resection. It increases the percentage of total removal of the tumor (12). According to the above results, we want to demonstrate for the first time a case of penile-scrotal EMPD was treated effectively by Mohs surgery resection guided by 5-ALA fluorescence.

This study allowed us to examine the usefulness of these techniques for detecting tumor border and distant scattered lesions. To evaluate the characteristics and efficacy of 5-ALA fluorescence-guided resection of EMPD, we report a clinical series of 5 patients during surgical resection of the lesions.

\section{Materials and Methods}

\subsection{Inclusive criteria}

According to the experience of the department of dermatology on the therapy in skin disease using the 5-ALA (13), the present study included 5 patients with penile-scrotal extramammary Paget's disease at our department between January 2014 and December 2015. All patients were diagnosed by biopsy in the department of dermatology and without prior chemotherapy ra $\neg$ diotherapy or biological therapy, or laser, frozen, or focal drug application.

\subsection{Exclusive criteria}

Those patients with severe light allergy, cardiac or cerebral vascular disease, severe liver or heart failure, other malignant tumor or hematologic disease, systemic immune disease, infectious disease, or inflammatory disease. Those patients who could not finish follow-up, died accidentally, or had incomplete clinical information.

\subsection{Major equipment and reagent}

The 5-aminolevulinic acid (5-ALA) (Shanghai Fudan-zhangjiang Bio-Pharmaceutical Co., Ltd. Shanghai, China) was obtained from the hospital. The photodynamic diagnosis instrument used the ultraviolet torch.

\subsection{Surgical procedures}

All patient's penile-scrotal lesions were coated with 5-ALA (concentration of $20 \%$ ) throughout the visible range of the lesion plus a $2 \mathrm{~cm}$ margin 3 hours before the induction of anesthesia. Surgical margin was determined in a standardized manner. The extent of resection was evaluated on the basis of frozen and histology sections. If the fluorescence positive punctate lesions were found outside the resection range, we removed the lesions and sent them for pathological examination. Each biopsy site was sutured with 4-0 nylon.

\subsection{Case Illustration}

A 72-year-old male patient with recurrent penilescrotal extramammary Paget's disease was initially admitted to the Urology Department of our hospital. Physical examination showed that there was sporadic edema erythema, popular eruption over an area of 6.0 $\times 3.0 \mathrm{~cm}^{2}$ in the penile-scrotal region (Figure $1 \mathrm{~A}$ ). Histopathological examination biopsy confirmed extramammary Paget's disease. We prepared a fresh solution of 5-aminolevulinic acid (5-ALA) solution $(20 \%)$ by dissolving 5 -ALA powder in physiological saline solution. The lesion plus a $2 \mathrm{~cm}$ margin were covered by ALA-soaked gauze for $3 \mathrm{~h}$ (Figure 1B). Before surgery, lesion locations were visualized by fluorescence examination using a light emitting diode (LED) emitting ultraviolet (UV) light at $405 \mathrm{~nm}$. 5-ALA's fluorescent product, protoporphyrin IX (PpIX), showed a characteristic bright brick-red color (Figure 1C). Under light visualization, the fluorescing areas were marked (Figures. 2A, 2B). Lesions in the blue light turn red after irradiation, the fluorescence-guided surgery delineated range is less than the naked eye can see, intraoperative frozen prompted negative margins, A total of 7 scattered lesions were found and resected (Figures 2C, 2D).

\subsection{Tumor pathology}

Tissue samples first underwent immediate frozen section analysis. After the operation all samples including scattered lesions were sent for routine histology.

\subsection{Postoperative complications and follow-up}

Follow-ups were performed every 3 months after treatment via telephone or outpatient clinic visits, and follow-up lasted for 12-24 months (average followup 17.8 months). Side-effects such as photosensitivity reactions or inflammation and recrudescence were observed.

\section{Results and Discussion}

Clinical and histological characteristics including 


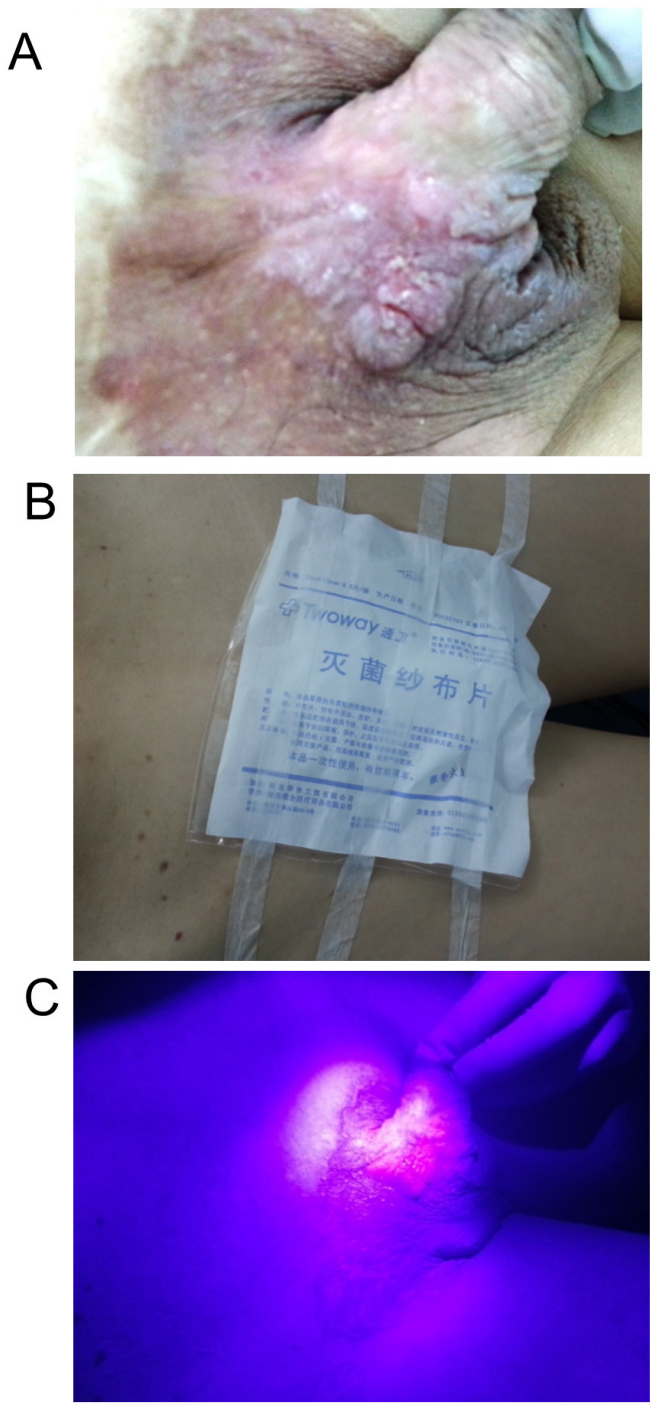

Figure 1. Surgical procedures. (A) A 72-year-old male patient with recurrence of penile-scrotal extramammary Paget's disease showed that there was sporadic edema erythema, popular eruption over an area $6.0 \times 3.0 \mathrm{~cm}^{2}$ in the penilescrotal region. (B) The penile-scrotal lesion was coated with 5-ALA (concentration of $20 \%$ ) throughout the visible range of lesion plus a $2 \mathrm{~cm}$ margin 3 hours before the induction of anesthesia. (C) Before surgery, lesion locations were visualized by fluorescence examination using a light emitting diode (LED) emitting ultraviolet (UV) light at $405 \mathrm{~nm}$. Under light visualization, the fluorescing areas were marked. Lesions in the blue light turned red after irradiation.

surgical results are summarized in Table 1. Five patients with penile-scrotal extramammary Paget's disease (all men, median age, 69.8 years; range, $65-77$ years) were enrolled in the analysis. Tumors were diagnosed based on biopsy by department of dermatology. The mean maximal tumor diameter of all surgical specimens was $5.6 \mathrm{~cm}$ (range, $4-7 \mathrm{~cm}$ ) (pre-PDD) and $4.8 \mathrm{~cm}$ (range, $3.5-6 \mathrm{~cm}$ ) (post-PDD). All 5 patients underwent the surgical procedures described above, and no sideeffects were attributed to 5-ALA. Lesions in the blue light turn red after irradiation, the fluorescence-guided surgery delineated range is less than the naked eye can see, intraoperative frozen sections prompted negative margins, for a postoperative pathological diagnosis. A total of 31 scattered lesions were found. After serial biopsies pathology prompted that four were positive. Surgical margins were negative in all patients.

The median follow-up duration was 17.8 months (range, 12-24 months). All patients were alive with no evidence of side-effects (photosensitivity reaction or inflammation) and local recurrence or metastasis at the end of the follow-up period.

Extramammary Paget disease (EMPD) is a cutaneous neoplasm presenting with erythematous spread, mainly on the genitals. As the tumor margin sometimes presents as ill-defined and multicentric, local recurrence after surgical excision is an important issue to overcome. Although surgical treatment with wide local excision (WLE) has been the standard choice of treatment, the recurrence rate is reported to be $33-60 \%$ (14). The significant morbidity and deformity after WLE is also problematic.

Differentiation of EMPD of the penoscrotal area from normal tissue is clearly frequently difficult, and setting a clear surgical margin is also difficult because of scrotal tissue redundancy. Therefore, various trials, such as intraoperative frozen sections or Mohs micrographic surgery (MMS), were performed to achieve tumor-free margins. However, frozen sections have a false-negative rate of $10.4 \%$, up to as high as $40 \%$, and is known to have no improvement in disease outcome $(15,16)$. MMS may lower local recurrence rate (17-20) but is timeconsuming and requires additional faculty for use.

As the tumor margin sometimes presents as illdefined and multicentric, local recurrence after surgical excision is an important issue to overcome. Photodynamic diagnosis has been utilized intraoperatively to detect positive surgical margins (21). Over the last decade, 5-aminolevulinic acid (5-ALA) has acquired considerable attention as a feasible agent for photodynamic diagnosis and therapy.

ALA is a precursor to heme in the heme cycle. When excess ALA is supplied to tissues, malignant tumor cells can take up the exogenous 5-ALA and convert it to a fluorescent photosensitizer, protoporphyrin IX (PpIX). Monitoring the distribution of PpIX is a useful tool for visualizing tumor locations, since the 5-ALA-induced PpIX fluorescence accumulates primarily in malignant tumor tissue and its fluorescence is much stronger than that in normal skin (22).

This report demonstrates for the first time the effective treatment of penile-scrotal extramammary Paget's disease with Mohs surgery resection guided by 5-ALA-induced fluorescence. To define clinically the apparent borders of the tumor as well as the extent of the tumor invasiveness 5-ALA was used. Biopsies from all the fluorescing lesions showed some histological extramammary Paget's disease, indicating the efficiency of ALA-induced fluorescence in detection and demarcation of EMPD. 

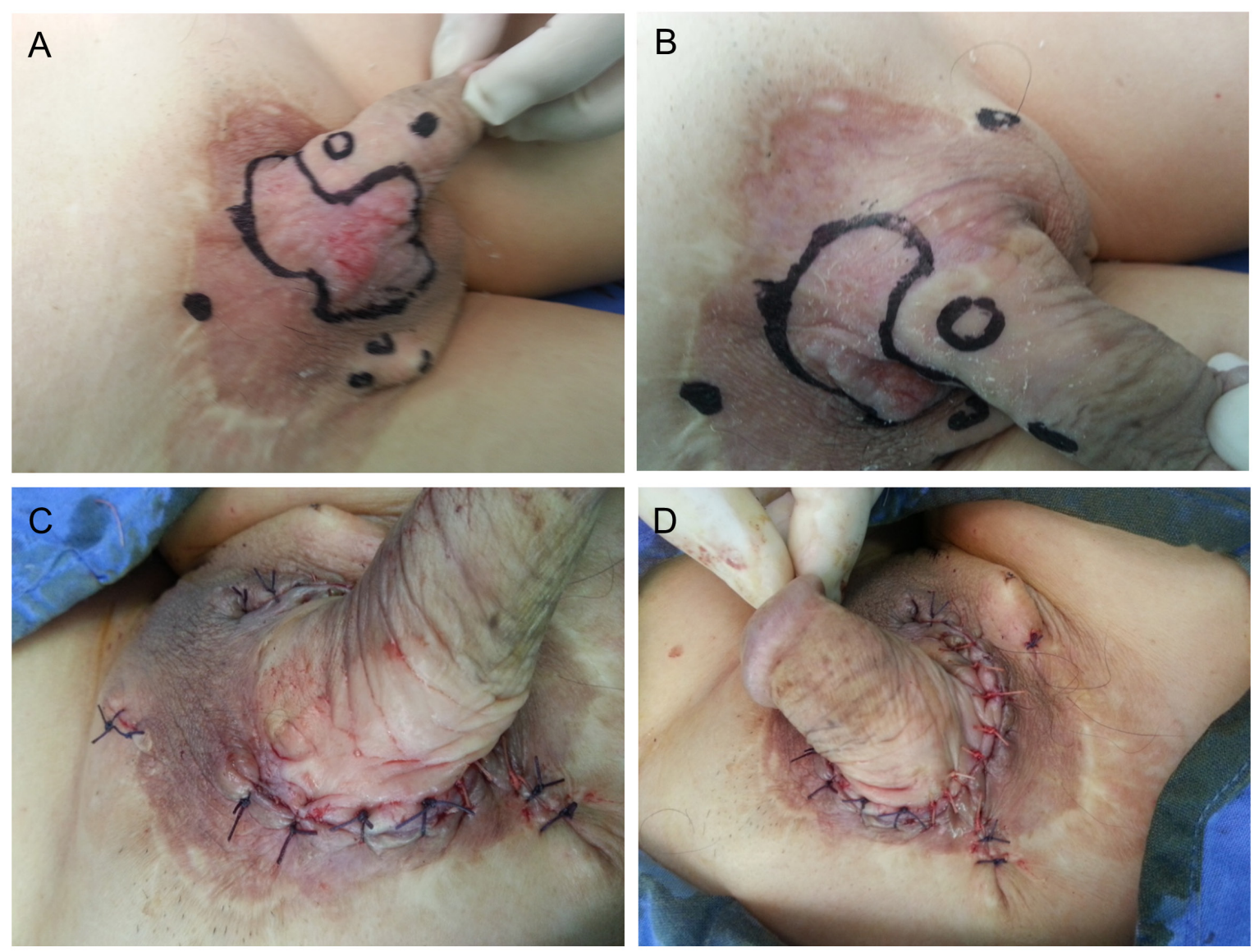

Figure 2. Lesions in the blue light turns red after irradiation, the fluorescence-guided surgery delineated range is less than the naked eye can see (A, B), intraoperative frozen sections prompted negative margins, scattered lesions were found and resected $(C, D)$.

Table 1. Surgical characteristics, pathological findings, short and long-term outcomes of patients with penile-scrotal extramammary Paget's disease

\begin{tabular}{lcccccc}
\hline No. & Age (yr) & Biopsy & $\begin{array}{c}\text { Tumor size }(\mathrm{cm}) \\
\text { Pre-PDD }\end{array}$ & $\begin{array}{c}\text { Tumor size (cm) } \\
\text { Post-PDD }\end{array}$ & $\begin{array}{c}\text { Scattered lesions } \\
\text { (PDD+) }\end{array}$ & Pathology positive \\
\hline 1 & 67 & Paget & $5.0 \times 6.0$ & $4.0 \times 5.0$ & 7 & 1 \\
2 & 65 & Paget & $5.0 \times 6.5$ & $5.0 \times 6.0$ & 6 & 0 \\
3 & 69 & Paget & $4.0 \times 5.5$ & $5.0 \times 6.0$ & 5 & 0 \\
4 & 77 & Paget & $5.0 \times 7.0$ & $4.5 \times 5.0$ & 7 & 2 \\
5 & 71 & Paget & $5.0 \times 6.5$ & $3.5 \times 4.0$ & 6 & 16 \\
\hline
\end{tabular}

Protoporphyrin seems to be the metabolite mainly responsible for tissue fluorescence. Slight fluorescence is always present in normal skin especially that of the intertriginous areas. This is due to the normal presence of propionibacteria, which are able to produce porphyrins (23). The fluorescence of these bacteria is pale and ill defined. In addition, after ALA application, normal unaffected skin of any area is also able to produce porphyrins, showing a slightly ill defined fluorescence. In contrast, the fluorescence of the tumor tissue is intense and sharply demarcated. When using 5-aminolevulinic acid (5-ALA)-mediated Photodynamic diagnosis (PDD), many studies have obtained better results (24-28).

However, in some cases it is difficult to excise the lesion completely, especially if it is distant or located in a critical anatomical site. In this study, we revealed feasibility of photodynamic diagnosis using 5-ALA for scattered lesions or those located in a critical anatomical site resection by direct intraoperative inspection of the tumor. We showed that the use of 5-ALA led to a higher frequency of complete resection of the scattered lesions and avoided large-scale resections. Intraoperatively, 5-ALA fluorescence-guided resection was useful especially when a tumor was distant or located in a critical anatomical site.

The study had some limitations such as small sample size and a short follow-up period. However, our results indicate that 5-ALA fluorescence-guided resection of penile-scrotal extramammary Paget's lesions was a 
feasible strategy for visualizing margins and maximum tumor resection was achieved with satisfactory outcomes.

In conclusion 5-ALA fluorescence-guided minimum range can completely remove penile-scrotal extramammary Paget's lesions, and it is able to detect distant scattered lesions.

\section{References}

1. Crocker HR. Paget's disease affecting the scrotum and penis. Trans Pathol Soc London. 1889; 40:187-191.

2. Zollo JD, Zeitouni NC. The Roswell Park Cancer Institute experience with extramammary Paget's disease. Br J Dermatol. 2000; 142:59-65.

3. Hendi A, Brodland DG, Zitelli JA. Extramammary Paget's disease: Surgical treatment with Mohs' micrographic surgery. J Am Acad Dermatol. 2004; 51:767-773.

4. Nardelli AA, Stafinski T, Menon D. Effectiveness of photodynamic therapy for mammary and extra-mammary Paget's disease: A state of the science review. BMC Dermatol. 2011; 11:13.

5. Cohen PR, Schulze KE, Tschen JA, Hetherington GW, Nelson BR. Treatment of extramammary Paget disease with topical imiquimod cream: Case report and literature review. South Med J. 2006; 99:396-402.

6. Thunshelle C, Yin R, Chen Q, Hamblin MR. Current Advances in 5-Aminolevulinic Acid Mediated Photodynamic Therapy. Curr Dermatol Rep. 2016; 5:179190.

7. Lv T, Zhang JC, Miao F, Wang HW. Aminoleveulinate photodynamic therapy (ALA-PDT) for Bowen's disease in a SLE patient: Case report and literature review. Photodiagnosis Photodyn Ther. 2017; 18:20-23.

8. Ang JM, Riaz IB, Kamal MU, Paragh G, Zeitouni NC. Photodynamic Therapy and Pain: A Systematic Review. Photodiagnosis Photodyn Ther. 2017; 19:308-344.

9. Hou J, Zhang Q, Fujino M, Cai S, Ito H, Takahashi K, Abe F, Nakajima M, Tanaka T, Xu J, Zou H, Ding Q, Li XK. 5-Aminolevulinic acid with ferrous iron induces permanent cardiac allograft acceptance in mice via induction of regulatory cells. J Heart Lung Transplant. 2015; 34:254-263.

10. Fujino M, Nishio $Y$, Ito $H$, Tanaka T, Li XK. 5-Aminolevulinic acid regulates the inflammatory response and alloimmune reaction. Int Immunopharmacol. 2016; 37:71-78.

11. Stummer W, Pichlmeier U, Meinel T, Wiestler OD, Zanella F, Reulen HJ. Fluorescence-guided surgery with 5-aminolevulinic acid for resection of malignant glioma: A randomized controlled multicentre phase III trial. Lancet Oncol. 2006; 7:392-401.

12. Chan DTM, Yi-Pin Sonia H, Poon WS. 5-Aminolevulinic acid fluorescence guided resection of malignant glioma: Hong Kong experience. Asian J Surg. 2017; pii: S10159584(17)30247-6.

13. Zhang Z, Lu XN, Liang J, Tang H, Yang YS, Zhu XH, Du J, Shen YY, Xu JH. Evaluation of photodynamic therapy using topical aminolevulinic acid hydrochloride in the treatment of condylomata acuminate. Int J Clin Exp Med. 2015; 8:6517-6521.

14. Lam C, Funaro D. Extramammary Paget's disease: Summary of current knowledge. Dermatol Clin. 2010; 28:807-826.
15. Gunn RA, Gallager HS. Vulvar Paget's disease: A topographic study. Cancer. 1980; 46:590-594.

16. Chan JYW, Li GKH, Chung JHP, Chow VLY. Extramammary Paget's disease: 20 years of experience in Chinese population. Int J Surg Oncol. 2012; 2012:1-5.

17. O'Connor WJ, Lim KK, Zalla MJ, Gagnot M, Otley CC, Nguyen TH, Roenigk RK. Comparison of Mohs micrographic surgery and wide excision for extramammary Paget's disease. Dermatol Surg. 2003; 29:723-727.

18. Lee KY, Roh MR, Chung WG, Chung KY. Comparison of Mohs micrographic surgery and wide excision for extramammary Paget's Disease: Korean experience. Dermatol Surg. 2009; 35:34-40.

19. Bae JM, Choi YY, Kim H, Oh BH, Roh MR, Nam K, Chung KY. Mohs micrographic surgery for extramammary Paget disease: A pooled analysis of individual patient data. J Am Acad Dermatol. 2013; 68:632-637.

20. Kim SJ, Thompson AK, Zubair AS, Otley CC, Arpey CJ, Baum CL, Roenigk RK, Lohse CM, Brewer JD. Surgical Treatment and Outcomes of Patients With Extramammary Paget Disease: A Cohort Study. Dermatol Surg. 2017; 43:708-714

21. Thunshelle C, Yin R, Chen Q, Hamblin MR. Current Advances in 5-Aminolevulinic Acid Mediated Photodynamic Therapy. Curr Dermatol Rep. 2016; 5:179190.

22. Yang X, Palasuberniam P, Kraus D, Chen B. Aminolevulinic Acid-Based Tumor Detection and Therapy: Molecular Mechanisms and Strategies for Enhancement. Int J Mol Sci. 2015; 16:25865-25880.

23. Flohil SC, van Lee CB, Beisenherz J, Mureau MA, Overbeek LI, Nijsten T, van den Bos RR. Mohs micrographic surgery of rare cutaneous tumours. J Eur Acad Dermatol Venereol. 2017; 31:1285-1288.

24. Wang W, Tabu K, Hagiya Y, Sugiyama Y, Kokubu Y, Murota Y, Ogura SI, Taga T. Enhancement of 5-aminolevulinic acid-based fluorescence detection of side population-defined glioma stem cells by iron chelation. Sci Rep. 2017; 7:42070.

25. Inoue Y, Imai Y, Fujii K, Hirokawa F, Hayashi M, Uchiyama $\mathrm{K}$. The utility of 5-aminolevulinic acidmediated photodynamic diagnosis in the detection of intraoperative bile leakage. Am J Surg. 2017; 213:10771082.

26. Hillemanns P, Wimberger P, Reif J, Stepp H, Klapdor R. Photodynamic diagnosis with 5-aminolevulinic acid for intraoperative detection of peritoneal metastases of ovarian cancer: A feasibility and dose finding study. Lasers Surg Med. 2017; 49:169-176.

27. Kishi K, Fujiwara Y, Yano M, Motoori M, Sugimura K, Takahashi H, Ohue M, Sakon M. Usefulness of diagnostic laparoscopy with 5-aminolevulinic acid (ALA)-mediated photodynamic diagnosis for the detection of peritoneal micrometastasis in advanced gastric cancer after chemotherapy. Surg Today. 2016; 46:1427-1434.

28. Motoori M, Yano M, Tanaka K, Kishi K, Takahashi H, Inoue M, Saito T, Sugimura K, Fujiwara Y, Ishikawa $\mathrm{O}$, Sakon M. Intraoperative photodynamic diagnosis of lymph node metastasis in esophageal cancer patients using 5-aminolevulinic acid. Oncol Lett. 2015; 10:3035-3039.

(Received August 29, 2017; Revised October 5, 2017; Accepted October 9, 2017) 